\title{
Heuristic UTD Coefficients Applied for the Channel Characterization in an Andean Scenario
}

\author{
Diego Tami, Cássio G. Rego, Fernando J. S. Moreira \\ Graduate Program in Electrical Engineering \\ Federal University of Minas Gerais \\ Belo Horizonte, MG, Brazil \\ \{diegotami; fernandomoreira\}@ufmg.br, \\ cassio@cpdee.ufmg.br
}

\author{
Dinael Guevara \\ Electrical and electronic Department \\ Universidad Francisco de Paula Santander \\ Cúcuta, N. Santander, Colombia \\ dinaelgi@ufps.edu.co
}

\author{
Andrés Navarro \\ ICT Department \\ Universidad Icesi \\ Cali, Valle, Colombia \\ anavarro@icesi.edu.co
}

\begin{abstract}
This paper presents a comparison of three heuristic coefficients for the Uniform Theory of Diffraction (UTD), used to characterize the radiowave scattering in typical urban scenarios. The coefficients were implemented in a propagation model based on 3D ray-tracing techniques in an andean scenario. In order to evaluate each coefficient we analyze the statistical behavior of the mean and standard deviation of the absolute errors between the estimated values and the measured data of path loss in a large number of receptor points. Finally, we show the path loss prediction for each heuristic UTD coefficients proposed.
\end{abstract}

\section{INTRODUCTION}

The continued development of wireless technologies, particularly in urban environment, leads to investigate methods to estimate, with high precision, the propagation parameters of wide-band channels in order to minimize the error with respect to on-site measurements. In recent years, methods based on ray tracing and UTD have shown accuracy and efficiency in the simulation of path-loss in complex environments. This accuracy depends mainly on the ray physical model in realistic environments and the numerical model used for estimating the scattered field. Therefore, the choice of the diffraction coefficients is important to accurately predict the signal amplitude obtained from the diffraction process.

Luebbers was one of the first researchers that established heuristic diffraction coefficients for lossy conducting wedges [1]. Luebbers' contributions have triggered a large number of studies to improve the accuracy of the heuristic coefficients. Among the most recent researches, Schettino et al [2] proposed heuristic UTD coefficients that ensuring reciprocity and providing superior performance in arbitrary source and observer locations. Guevara et al [3] used a physical technique that model the edge where diffraction occurs to obey reciprocity and adopt two types of permittivity to characterize the building materials. This paper will present the implementation of these three heuristic UTD coefficientsin a $3 \mathrm{D}$ ray-tracing model in order to evaluate the precision of the model with respect to measurements in a realistic scenario that has been previously validated in [4].

\section{DESCRIPTION AND SiMULATION OF THE PROPAGATION CHANNEL}

This section describes the heuristic UTD coefficients used for the comparison, the outdoor scenario and the measurement campaign. Finally, it shows the analysis and results of the path loss prediction using the 3D ray-tracing model.

\section{A. Heuristic UTD Coefficients}

The UTD electric field at the observer is defined as [1]:

$$
E_{d}(O)=E_{i}(W) \cdot \bar{D} \cdot A\left(s_{d}\right) \cdot e^{-j k s_{d}}
$$

where $E_{i}(W)$ is the incident electric field at the wedge, $A\left(s_{d}\right)$ is the amplitude factor, $s_{d}$ is the distance between wedge and observer, and $\bar{D}$ is the dyadic diffraction coefficient. This paper implements three heuristic coefficients used for the characterization of the radio channel:

1) Luebbers' coefficients [1]: It introduced the Fresnel reflection coefficients in the UTD diffraction coefficients, defining incidence and reflection angles according to the incident and diffracted rays. However, it presents difficulties associated with reciprocity and deep shadow regions.

2) Schettino's coefficients [2]: It proposed a heuristic UTD coefficients mainly based on Holm's formulation [5], with angular definitions for $\alpha_{0}$ and $\alpha_{n}$ based on [6]. It ensures reciprocity and providing superior performance in arbitrary source and observer locations.

3) Guevara's coefficients [3]: It based in [1] and the application of a physical technique in order to obey reciprocity and specification of permittivity of the building materials. This technique allows modeling the diffraction from the top and side edges of each building.

\section{B. Outdoor Scenario}

The simulated and analyzed scenario was an urban microcell in the downtown of Cali, Colombia [4]. Figure 1 shows the 3D urban model; we used the Cost2100 Cali Realistic Scenario with $1 \mathrm{~m}$ resolution. This scenario has a 
$512 \mathrm{~m} \times 512 \mathrm{~m}$ area, with strongly tilted roads and complex building architecture of different heights. We implemented a 3D model using spatial geometries for representing streets, roofs and walls of buildings. Most of the buildings and streets materials in the selected area of the city have the same constitutive parameters. Specifically, we assumed brick ( $\varepsilon r=7-$ $\mathrm{j} 0.3, \mu \mathrm{r}=1$ ) for all building walls; a first type of dry concrete ( $\varepsilon r=5.3-\mathrm{j} 0.25, \mu \mathrm{r}=1)$ for all building roofs; and a second type of dry concrete $(\varepsilon r=7-j 0.3, \mu r=1)$ for street pavement, these parameters are permittivity optimized values mentioned in[7].

\section{Path Loss Measurements campaign}

The measurements campaign consisted in collecting the receivedpowerin a specific route in the streets around the transmitter (see green spheres in Fig. 1). The transmitter antenna is a vertically polarized omni-directional antenna, with a gain of $6.5 \mathrm{dBi}$, it provided a signal at $900 \mathrm{MHz}$ and it's located on the top of the building at $16 \mathrm{~m}$ above this (see blue object in Fig. 1). The receiver system consists of a mobile low profile vertically polarized antenna, with a measured gain of 3 $\mathrm{dBi}$. This system is mounted in the test car, with the receiver antenna positioned above the center of the rooftop of the vehicle, which corresponds to an antenna height of $2.1 \mathrm{~m}$ above ground. The acquisition time was $1 \mathrm{~ms}$. The radio channel measurement characterized the propagation loss in the selected route, which include localizations with LOS and NLOS. It was obtained in total 258 measurement points for the drive test.

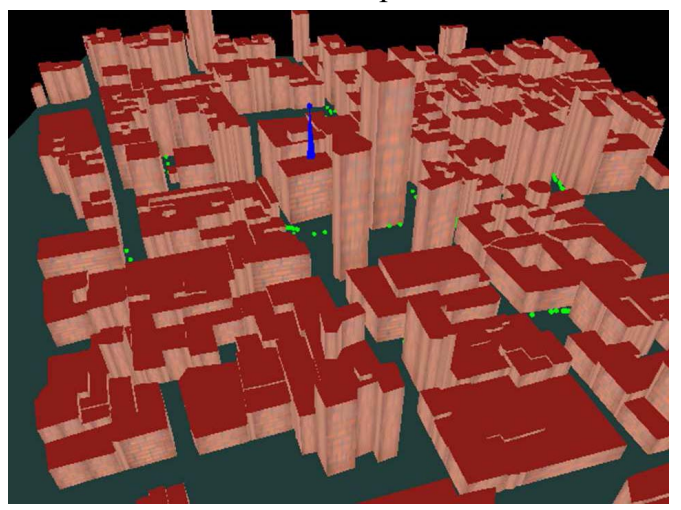

Fig. 1.The 3D urban model for Cali using the game engine.

\section{Analysis and Results}

Figures 2, 3 and 4 show the path loss prediction at the receiver locations, where results obtained by the heuristic UTD coefficients presented in Section II are compared with measurements. The comparisons show that the Luebbers' coefficients present the more variable response with respect to measurement. Furthermore, Guevara's and Schettino's coefficients present a close response, thus the Figs. 3 and 4 show an approximate prediction in some points of reception. In order to evaluate which one is more accurate, we calculate the mean and standard deviation of the absolute errors. These statistical results are summarized in Table I. Statistical analysis shows that for this scenario, Guevara's coefficients [5] provide slightly better results, with a standard deviation of $5.36 \mathrm{~dB}$, predicted value indicating high accuracy; and a mean absolute error of $11.25 \mathrm{~dB}$. However, the statistical difference with respect other heuristic formulations $[1,2]$ is small.

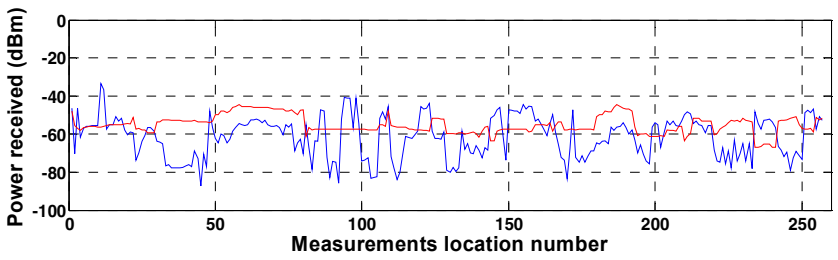

Fig. 2.Power prediction comparison between ray tracing using Luebbers' coefficients (blue line) and measurements (red line).

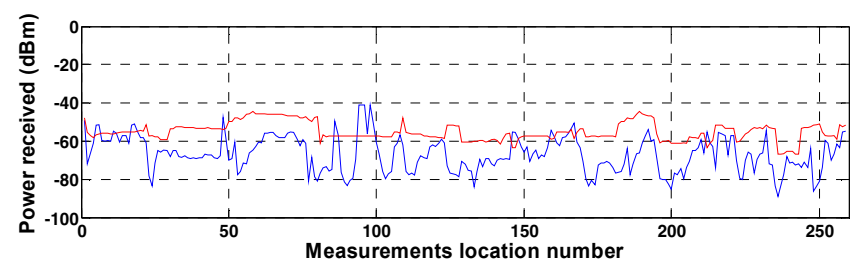

Fig. 3.Power prediction comparison between ray tracing using Schettino et al coefficients (blue line) and measurements (red line).

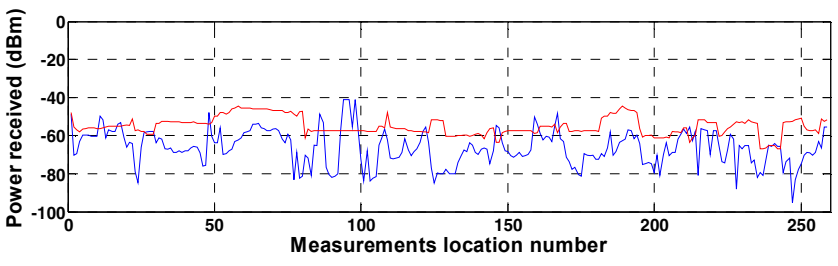

Fig. 4.Power prediction comparison between ray tracing using Guevara et al coefficients (blue line) and measurements (red line).

Table I. Statical Summary of Path Loss Prediction

\begin{tabular}{|l|c|c|}
\hline \multicolumn{1}{|c|}{ UTD Coefficients } & $\begin{array}{c}\text { Mean absolute error } \\
(\mathbf{d B})\end{array}$ & $\begin{array}{c}\text { Standard Deviation } \\
(\mathbf{d B})\end{array}$ \\
\hline Luebbers & 8.76 & 5.75 \\
\hline Schettino & 11.73 & 5.56 \\
\hline Guevara & 11.25 & 5.36 \\
\hline
\end{tabular}

\section{ACKNOWLEDGEMENT}

This work was supported by CNPq and CAPES, Brazil.

\section{REFERENCES}

[1] R. Luebbers, "A heuristic UTD slope diffraction coefficient for rough lossy wedges," IEEE Trans. Antennas Propagat., vol. 37, no. 2, pp. 206211, February 1989.

[2] D. Schettino, F. Moreira, and C. Rego, "Heuristic UTD coefficients for electromagnetic scattering by lossy conducting wedges, " Wiley Per., Inc. Microwave Opt Technol Lett., vol. 52, no. 12, pp. 2657-2662, Dec 2010.

[3] A. Navarro, D. Guevara, N. Cardona, and J. Gimenez, "DVB Coverage prediction using Game Engine based Ray-Tracing Techniques, ” in IEEE 74th Veh. Tech. Conf. VTC 2011-Fall, San Francisco, USA, Sep 2011.

[4] A. Navarro and D. Guevara, "Applicability of game engine for ray tracing techniques in a complex urban environment," in IEEE 72nd Veh.Tech. Conf. VTC 2010-Fall, Ottawa, Canada, September 2010.

[5] P. Holm, "A new heuristic UTD diffraction coefficient for nonperfectly conducting wedges," IEEE Trans. Antennas Propagat., vol. 48, no. 8, pp. 1211-1219, August 2000.

[6] H. El-Sallabi and P. Vainikainen, "Improvements to diffraction coefficient for non-perfectly conducting wedges," IEEE Trans. Antennas Propagat., vol. 53, no. 9, pp. 3105-3109, September 2005.

[7] A. Navarro, D. Guevara, N. Cardona, and J. Lopez, "Measurement-based ray-tracing models calibration in urban environments," in IEEE Antennas and Propag. Soc. Inter. Symp. (APS/URSI), Chicago, USA, July 2012. 